\title{
A NOTE ON MULTIVALENT STARLIKE FUNCTIONS
}

A. W. GOODMAN AND J. A. HUMMEL 1

In a recent paper [2], G. R. Blakley raised a question about the decomposition of a $p$-valent starlike function into the product of two $p$-valent starlike functions. It is the purpose of this note to prove the conjecture of Blakley.

Definition 1. The function $f(z)$, regular in $|z|<1$, is said to be in the class $S(p, m)$, where $p$ and $m$ are positive integers with $p \geqq m$, if and only if

(1) there exists a positive $\rho<1$ such that

$$
\operatorname{Re}\left\{\frac{z f^{\prime}(z)}{f(z)}\right\}>0, \quad \rho<|z|<1,
$$

$$
\begin{gathered}
f(z)=z^{m}+a_{m+1} z^{m+1}+\cdots, \quad|z|<1, \\
\int_{0}^{2 \pi} \operatorname{Re}\left\{\frac{z f^{\prime}(z)}{f(z)}\right\} d \theta=2 \pi p, \quad z=r e^{i \theta}, \rho<r<1
\end{gathered}
$$

(i.e., $f(z)$ is $p$-valent in $|z|<1$ ). In other words, $f(z)$ belongs to $S(p, m)$ if and only if $f(z)$ is $p$-valently starlike with respect to the origin and has an $m$ th order zero at $z=0$.

Let us use the convention that, if $A$ and $B$ are two sets of functions, then

and

$$
A B=\{f(z) \mid f(z)=g(z) h(z), g \in A, h \in B\}
$$

$$
A^{n}=\left\{f(z) \mid f(z)=[g(z)]^{n}, g \in A\right\} .
$$

With this notation we then have

$$
S(p, p)=[S(1,1)]^{p}
$$

as has been proved by Robertson [8]. The conjecture of Blakley then becomes

THEOREM 1. If $1<m<p$, then

$$
\begin{aligned}
S(p, m) & =S(m-1, m-1) S(p-m+1,1) \\
& =[S(1,1)]^{m-1} S(p-m+1,1) .
\end{aligned}
$$

Received by the editors November 23, 1963.

${ }^{1}$ The research of Professor Hummel was supported by the National Science Foundation under NSF-G 24469 during the preparation of this paper. 
The second expression in this theorem follows from the first because of the above-mentioned result of Robertson. It should be noted that there is an obvious error in the conjecture as printed in [2].

Here, a function $f(z)$ in $S(p, m)$ is decomposed into the product of two starlike functions, one of which has all of the nonzero zeros. This result should be compared with Theorems 2.1 and 3.1 of the recent paper of Bender [1].

The proof of Theorem 1 is accomplished with the help of a representation theorem of Paatero [7]. It may be remarked that the assignment of priority for this representation theorem is not at all certain. Similar types of formulas are found in several other references. A paper by Dundučenko [3] hints that a similar or related formula is contained in the Ph.D. thesis of Zmorovich, but this reference is not available to us. Paatero himself refers to an article by Komatu [5], and Maxsimov [6] develops and uses a similar type of formula.

Since this representation formula can be viewed as a generalization of the Schwarz-Christoffel transformation (the paper of Goodman [4] should be consulted in this regard) and is proved by fairly direct application of the Poisson-Stieltjes formula, it is not unlikely that similar formulas may have been developed earlier in papers which we are not aware of at this time.

The theorem as given by Paatero is directly applicable to our problem. We need, however, only a slightly weaker version of Paatero's theorem and, in stating the theorem, we delete the branch points at infinity which Paatero assumes may be present.

TheOREM A (PAATERo $[7, p .10])$. Let $G$ be a region of bounded boundary-turning $\alpha$ with finite branch points at $A_{1}, A_{2}, \cdots, A_{n}$ of orders $\mu_{1}, \mu_{2}, \cdots, \mu_{n}$. Let $\zeta=\zeta(z)$ map $|z|<1$ conformally on $G$ with $a_{1}, a_{2}, \cdots, a_{n}$ going into $A_{1}, A_{2}, \cdots, A_{n}$.

Set $f(z)=u(z)+i v(z)=1+z \zeta^{\prime \prime}(z) / \zeta^{\prime}(z)$. Then the limit

$$
\psi_{1}(\theta)=\lim _{r \rightarrow 1} \int_{0}^{\theta} u\left(r e^{i \phi}\right) d \phi
$$

exists for $0 \leqq \theta \leqq 2 \pi$, except for a countable set of values of $\theta$. The function $\psi_{1}(\theta)$ is of bounded variation $\alpha$ and, further,

$$
\begin{aligned}
\zeta(z)= & C_{1} \int_{0}^{z} \prod_{k=1}^{n}\left[\left(1-\bar{a}_{k} z\right)\left(z-a_{k}\right)\right]^{\mu_{k}} \\
& \cdot \exp \left\{-\frac{1}{\pi} \int_{0}^{2 \pi} \log \left(1-z e^{i \theta}\right) d \psi_{1}(\theta)\right\} d z+C_{2},
\end{aligned}
$$




$$
\int_{0}^{2 \pi} d \psi_{1}(\theta)=2 \pi\left[1+\sum_{k=1}^{n} \mu_{k}\right]
$$

Conversely, if $a_{1}, a_{2}, \cdots, a_{n}$ are arbitrary $\left(\left|a_{k}\right|<1\right)$ and $\psi_{1}(\theta)$ is an arbitrary function of bounded variation $\alpha$ which satisfies (6), then (5) maps $|z|<1$ onto a region whose boundary turning is $\alpha$, and which has, at the image points of $a_{k}$, branch points of order $\mu_{k}$.

If we restrict ourselves to convex $p$-valent functions $\zeta(z)$, then the image domain will have boundary turning $\alpha=2 \pi p$ and the function $\psi_{1}(\theta)=\psi(\theta)$ will be a monotone increasing function. This may be done by making use of the Alexander technique of associating a convex function $\zeta(z)$ with a starlike function $g(z)$ by means of the relation $g(z)=z \zeta^{\prime}(z)$ so that

$$
\frac{z g^{\prime}(z)}{g(z)}=1+\frac{z \zeta^{\prime \prime}(z)}{\zeta^{\prime}(z)}=f(z)
$$

Theorem A then will give us a representation theorem for starlike $p$-valent functions.

From this theorem we can thus conclude that $g(z)$ will be a $p$ valent starlike function if and only if there exists a monotone increasing function $\psi(\theta)$ of total variation $2 p \pi$ in the interval $[0,2 \pi]$ such that

$$
\begin{aligned}
g(z)= & C z \exp \left\{-\frac{1}{\pi} \int_{0}^{2 \pi} \log \left(1-z e^{-i \theta}\right) d \psi(\theta)\right\} \\
& \cdot \prod_{k=1}^{n}\left[\left(1-\bar{a}_{k} z\right)\left(z-a_{k}\right)\right]^{\mu_{k}} .
\end{aligned}
$$

In this representation, $\sum \mu_{k}=p-1$. If we let $m-1$ of the $a_{k}$ be zero and count the rest with their multiplicity, we have

$$
\begin{aligned}
g(z)= & C z^{m} \prod_{k=1}^{p-m}\left(1-\bar{a}_{k} z\right)\left(z-a_{k}\right) \\
& \cdot \exp \left\{-\frac{1}{\pi} \int_{0}^{2 \pi} \log \left(1-z e^{-i \theta}\right) d \psi(\theta)\right\} .
\end{aligned}
$$

The monotone function $\psi(\theta)$, as defined by (4), is such that $\psi(0)=0$ and $\psi(2 \pi)=2 \pi p$.

We now proceed to the proof of Theorem 1. If $g(z)$ is in $S(p, m)$, then we have the representation (8). We now merely need to decompose $\psi(\theta)$ into the sum of two monotone increasing functions $\psi_{1}(\theta)$ and $\psi_{2}(\theta)$ such that 


$$
\begin{aligned}
& \int_{0}^{2 \pi} d \psi_{1}(\theta)=2 \pi(m-1) \\
& \int_{0}^{2 \pi} d \psi_{2}(\theta)=2 \pi(p-m+1) .
\end{aligned}
$$

This can be done in infinitely many ways, but one obvious way is to set

$$
\psi_{1}(\theta)=\frac{(m-1)}{p} \psi(\theta),
$$

$$
\psi_{2}(\theta)=\frac{(p-m+1)}{p} \psi(\theta) .
$$

With this, or any other such decomposition, we then merely need to set

$$
\begin{aligned}
g_{1}(z)= & z^{m-1} \exp \left\{-\frac{1}{\pi} \int_{0}^{2 \pi} \log \left(1-z e^{-i \theta}\right) d \psi_{1}(\theta)\right\}, \\
g_{2}(z)= & C z \prod_{k=1}^{p-m}\left(1-\bar{a}_{k} z\right)\left(z-a_{k}\right) \\
& \cdot \exp \left\{-\frac{1}{\pi} \int_{0}^{2 \pi} \log \left(1-z e^{-i \theta}\right) d \psi_{2}(\theta)\right\},
\end{aligned}
$$

where

$$
C=(-1)^{p-m} / \prod_{k=1}^{p-m} a_{k}
$$

Then by Theorem A, $g_{1}(z)$ and $g_{2}(z)$ are in the classes $S(m-1, m-1)$ and $S(p-m+1,1)$, respectively, while clearly $g_{1}(z) g_{2}(z)=g(z)$. This completes the proof of Blakley's conjecture.

It may be observed that the above proof can be modified easily to obtain similar results. Indeed, it is clear that by allocating the zeros properly, we can prove:

THEOREM 2. If $1 \leqq m_{1} \leqq p_{1}, 1 \leqq m_{2} \leqq p_{2}$,

$$
S(p, m)=S\left(p_{1}, m_{1}\right) S\left(p_{2}, m_{2}\right)=S\left(p_{1}+p_{2}, m_{1}+m_{2}\right) .
$$

This result is of some interest with regard to the structure of the class of $p$-valent starlike functions. It is obvious that

$$
S\left(p_{1}, m_{1}\right) S\left(p_{2}, m_{2}\right) \subset S\left(p_{1}+p_{2}, m_{1}+m_{2}\right)
$$


since if $h=f g$, then $z h^{\prime} / h=z f^{\prime} / f+z g^{\prime} / g$. Theorem 2 shows that, in fact, equality holds in (10).

We see that a function in $S(p, m)$ can be decomposed in many ways into the product of functions in other classes. The decomposition of Theorem 1 gives us functions in $S(1,1)$ and a single function in $S(p-m+1,1)$. Another possible decomposition might be given when $m \geqq p / 2$. In this case, each nonzero zero would be paired with one zero and we would have

$$
S(p, m)=S(2,1) S(2,1) \cdots S(2,1) S(2 m-p, 2 m-p)
$$

where there are $p-m$ factors $S(2,1)$. The last factor would be missing if $p=2 m$.

The decomposition of a monotone function into the sum of two monotone functions is in no way unique. Thus, the decomposition of (9) cannot be considered as canonical. In particular it is clear that the functions mapping to domains bounded by radial slits do not play an extremal role with regard to this decomposition.

\section{REFERENCES}

1. John Bender, Some extremal theorems for multivalently star-like functions, Duke Math. J. 29 (1962), 101-106.

2. G. R. Blakley, Classes of p-valent starlike functions, Proc. Amer. Math. Soc. 13 (1962), 152-157.

3. L. E. Dunducenko, Certain extremal properties of analytic functions given in a circle and in a circular ring, Ukrain. Mat. Ž. 8 (1956), 377-395.

4. A. W. Goodman, On the Schwarz-Christoffel transformation and p-valent functions, Trans. Amer. Math. Soc. 68 (1950), 204-223.

5. Yûsaku Komatu, Einige Darstellungen analytischer Funktionen und ihre Anwendungen auf konforme Abbildung, Proc. Imp. Acad. Tokyo 20 (1944), 536-541.

6. D. Maxsimov, Estimation of coefficients for certain classes of analytic functions, Dokl. Akad. Nauk SSSR 110 (1956), 507-510.

7. V. Paatero, Über die konforme Abbildung mehrblättriger Gebiete von beschränkter Randdrehung, Ann. Acad. Sci. Fenn. Ser. A I 128 (1952), 1-14.

8. M. S. Robertson, The partial sums of multivalently star-like functions, Ann. of Math. (2) 42 (1941), 829-838.

UNIVERSITY OF KENTUCKY AND

UNIVERSITY OF MARYLAND 